\title{
Lung Ultrasound (in the Critically III) Superior to CT: the Example of Lung Sliding
}

\author{
Daniel A. Lichtenstein
}

Hôpital Ambroise-Paré (Medical ICU), Paris-Ouest university, Boulogne, France

This review article shows the potential of lung ultrasound in the critically ill (LUCI) to study lung sliding and describes the optimal equipment for its assessment. Then, it analyses the integration of lung sliding within lung ultrasound then whole body critical ultrasound. It describes the place of lung sliding in the BLUE-protocol (bedside lung ultrasound in emergency) (lung and venous ultrasound for diagnosing acute respiratory failure), the FALLS-protocol (fluid administration limited by lung sonography) (the role of lung sliding in circulatory failure), and the SESAME-protocol (sequential assessment of sonography assessing mechanism or origin of severe shock of indistinct cause) (whole body ultrasound in cardiac arrest). In the LUCIFLR project (LUCI favoring limitation of radiations), the consideration of lung sliding allows drastic reduction in irradiation and costs. In conclusion, lung sliding is proposed as a gold standard for indicating the presence of the lung at the chest wall and its correct expansion.

Key Words: acute respiratory failure; BLUE-protocol; critical ultrasound; lung sliding; lung ultrasound; pneumothorax.

\section{Introduction}

Ultrasound was since its birth an official tool for few disciplines: cardiology, gyneco-obstetrics, and radiology. By suggesting to add the most vital organ, in spite of the fact that its existence had be denied by the community [1], ultrasound - lung ultrasound - has become the tool of many specialties, critical care first. Some academicians still remain reluctant, in 2016. This review article will show, through one basic application (lung sliding) that lung ultrasound in the critically ill (LUCI) not only exists, but is able to be a gold standard in the critical settings and even beyond.

\section{What is Lung Sliding}

Due to the diaphragmatic contraction mainly, the lung inflates on inspiration, therefore descends toward the abdomen. This phenomenon, which occurs from birth to death without any interruption apart from voluntary apnea, can be visualized using ultrasound.

LUCI is a simple discipline, defined by seven principles, shortly reminded: (1) A simple machine is suitable. (2) The thorax

\author{
Received on November 22, 2016 Accepted on December 27, 2016 \\ Correspondence to: Daniel A. Lichtenstein, Hôpital Ambroise-Paré (Medical ICU), Paris-Ouest university, 9 avenue Charles de Gaulle, Boulogne, France \\ Tel: +33-1-49-09-56-01, E-mail: D.Licht@free.fr
}

*No potential conflict of interest relevant to this article was reported.

(cc) This is an Open Access article distributed under the terms of the Creative Commons Attribution Non-Commercial License (http://creativecommons.org/ licenses/by-nc/4.0/) which permits unrestricted non-commercial use, distribution, and reproduction in any medium, provided the original work is properly cited. Copyright (c) 2017 The Korean Society of Critical Care Medicine 
is an area where air and water live together, and the rules of gravity must apply. (3) The lung is the widest organ. (4) All arises from the pleural line. (5) The artifacts make the basis of LUCI. The artifacts clinically useful are the A-line and the B-line. (6) The lung is a vital organ, with a permanent dynamic: lung sliding (here detailed). LUCI is a dynamic discipline. (7) Almost all life-threatening disorders reach the chest wall (and are therefore detectable) to a large extent usually (and are therefore easily detected).

Lung sliding is one of the ten main signs of LUCI. Briefly, the first, the basis of all, is the pleural line. The A-line is a repetition of the pleural line indicating air (gas) below the pleural line, which indicates, always, the parietal pleura (Figure 1). Lung sliding (principle 6), on special focus here, indicates, briefly, that the pleural line is not only the parietal but also the visceral pleura. The quad sign indicates a pleural effusion, the sinusoid sign indicates its viscosity. The shred sign indicates a (non translobar, the most frequent) lung consolidation, the tissue-like sign is useful for diagnosing translobar cases. In

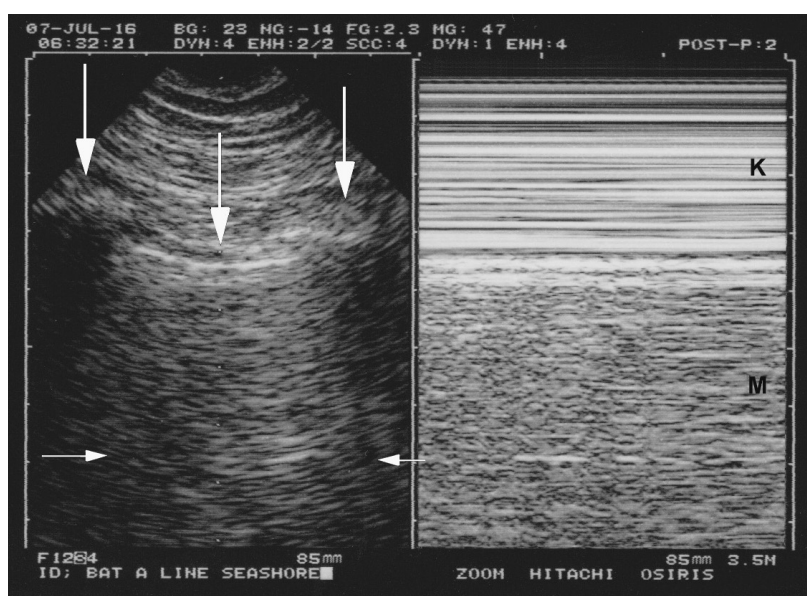

Figure 1. Normal lung sliding. Left, real time. Both ribs (lateral vertical arrows) and the pleural line (middle vertical arrow) outline the bat sign. In this adult, the pleural line is $0.5 \mathrm{~cm}$ below the rib line, allowing a confident recognition. Note one A-line partially visible (horizontal arrows) at the expected location (distance skin/pleural line). Right, M-mode. Lung sliding, not visible in the left (frozen) image, appears as a sandy pattern at and below the pleural line. This image shows two rectangular areas: from top to bottom, Keye's space (soft tissues) (K) and Merlin's space (the "lung") (M). These areas are strictly separated by the pleural line. Note that this smart technology (1992) displays the pleural line of the left and right images at the same level. the BLUE-protocol (bedside lung ultrasound in emergency), posterior lung consolidations and pleural effusions are gathered, for simplifying, since it does not change its accuracy, and the pattern is called posterolateral alveolar and/or pleural syndrome (PLAPS). The B-line is the elementary sign of interstitial syndrome. Figure 2 explains the B-line and lung rockets. The stratosphere sign, with the A-line sign, highly suggests pneumothorax. The lung point indicates pneumothorax. This makes ten signs. Some articles [2] or textbooks [3] go more into the detail. In the critical care, regarding pneumothorax, pleural effusion, lung consolidation and interstitial syndrome, lung ultrasound has an accuracy which places it not far from computed tomography (CT), the gold standard used in all our original articles. But how about lung sliding, how about its accuracy, which gold standard to use?

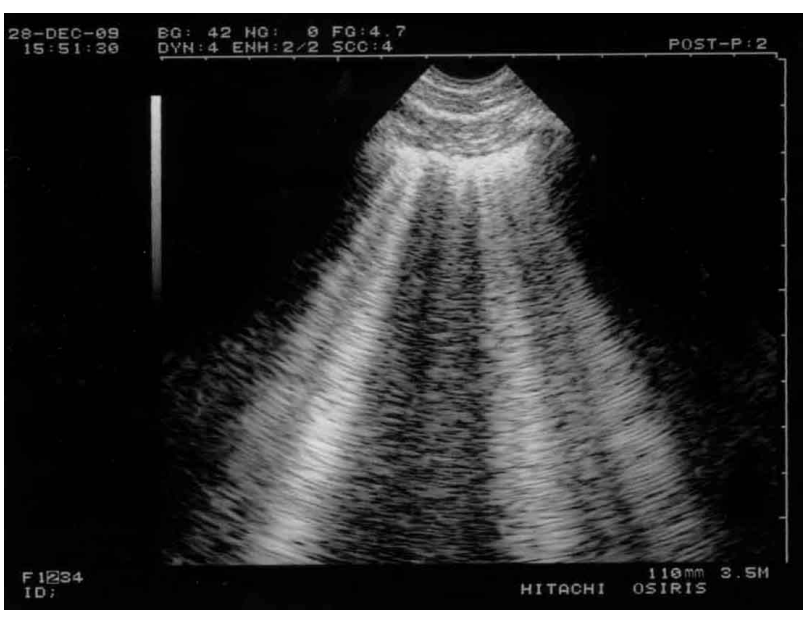

Figure 2. B-lines and lung rockets. Typical multiple B-lines. This figure shows the 7 features of the $B$-line. Three are constant. 1) A comet-tail artifact. 2) It always arises from the pleural line. 3) It always moves in concert with lung sliding (and is therefore motionless if lung sliding is abolished). A video of B-profile and B'profile is available at www.CEURF.net for this dynamic pattern. Four features are nearly always present: 4) long; 5) well-defined; 6 ) erasing A-lines; 7) and hyperechoicity. This definition allows recognition in any circumstances and avoids any confusion with other comet-tail artifacts. More than two B-lines between two ribs are called lung rockets, which are used to diagnose interstitial syndrome. Three or four B-lines are called septal rockets and correlate with edematous subpleural interlobular septa. The double, like here (at least seven) are called ground-glass rockets and correlate with computerized tomography ground glass areas. Diffuse lung rockets define the B-profile or the B'-profile (depending on the presence or absence of lung sliding). 


\section{Wich Equipment for an Efficient Study of Lung Sliding}

Before assessing lung sliding, we need first to define the tool. We use the principle $\mathrm{N}^{\circ} 1$ of LUCI: simplicity. The simplest machines, the oldest too, are the best for the control of lung sliding (as well as the whole of LUCI). Nowadays machines are equipped with filters, which enhance the image quality of static images at the detriment of the artifacts and the dynamics, two critical tools in LUCI. Providentially, some manufacturers have heard our message and begin to use again older technologies.

In practice, if we have to use a modern machine, we spend some time in finding, then by-passing all filters, mainly those which generate time lags, or alter artifacts. Dynamic noise filter, average filter, persistence, compound, harmonics are disactivated. We acquire lung ultrasound with natural images.

\section{Where to Search for a Basic Lung Sliding:} the BLUE-points

Before describing lung sliding, we need to show where to analyze it. Since the lung is the most voluminous organ, the question of where to apply the probe may appear challenging. In the BLUE-protocol (see below), we use three standardized points of analysis called the BLUE-points [4]. See Figure 3 for clinical use, we find the most convenient site for studying lung sliding is the lower BLUEpoint. At the apex, lung sliding can be analyzed using our microconvex probe, and is usually absent (the apex is the starting block of the lung's inspiratory descent).

One first detects the ribs, and then the pleural line, half a $\mathrm{cm}$ below the rib line in adults. We use a microconvex probe for this, because of its small footprint and many other advantages (ours is a universal one, allowing whole body analysis, a basic advantage in the extreme emergencies) (and low income settings) (also for limiting crossed infections).

We introduce the notion of Merlin's space and Keye's space. On real-time or M-mode, the space above the pleu-

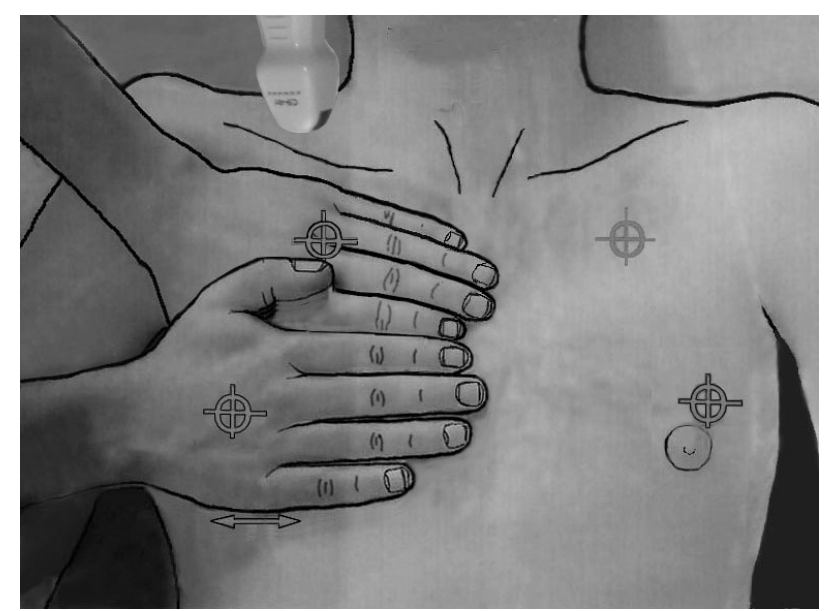

Figure 3. The BLUE-points. This image shows how the two hands (from the patient's size) are placed in order to produce a projection of the lung (the lower arrow indicates the usual location of the diaphragm). The upper point (upper BLUE-point) would show a discrete lung sliding. The lower point (lower BLUE-point) is the usual site for assessing the amplitude of lung sliding (maximal at the base). Lung sliding will be minimal at the upper BLUE-point and usually null at the apex (see how a microconvex probe can be inserted at the right apex). The PLAPS-point is the transversal continuation of the lower BLUEpoint, performed posteriorly to the posterior axillary line. BLUE: bedside lung ultrasound in emergency; PLAPS: posterolateral alveolar and/or pleural syndrome.

ral line has been called the Keye's space. The space limited by the pleural line, the shadow of ribs and the bottom of the screen is the Merlin's space. In M-mode, Keye's and Merlin's space are two superimposed rectangles (Figure 1).

\section{How Lung Sliding Appears in Healthy Sub- ject on Standard Conditions}

On inspiration, the visceral pleura slides against the motionless parietal pleura. On ultrasound, a longitudinal scan is the logical way to see this cephalo-podal dynamic.

On the screen, at the pleural line, a twinkling is observed (sparkling, shimmering, glittering are suitable names too). This twinkling is called lung sliding. It is seen from the pleural line and spreads homogeneously below, at the origin of the seashore sign (Figure 1). The seashore sign must appear from the pleural line, not one $\mathrm{mm}$ above, not one mm below. In other words, it must 
cover exactly the whole of the Merlin's space.

\section{What Can Now Be Inferred for the Physician Dealing with the Critically III}

Lung sliding is the obvious indicator of lung ampliation. One cannot describe any gold standard for such a dynamic. The physical examination is not precise enough (especially in a critically ill patient), the radiograph and even the CT do not provide dynamic data. The antique, irradiating fluoroscopy should be the nearest, but is not very precise, and anyway out of the reach of the critically ill. One must accept that lung ultrasound is the tool able to assess the lung excursion in clinical practice. One must accept to consider lung ultrasound as a gold standard.

In other words, lung sliding is able to allow physicians to better understand the very physiology of the lung [5].

How to assess the amplitude of lung sliding? This is easy in the case of any irregularity arising from the pleural line (called B-lines, I-lines, N-lines or C-lines, frequent in pathologic conditions, or some minute irregularities at the pleural line, seen in normal conditions, temporarily called Q-lines). If none is visible, more skill is required, but in this case, the probe can be placed at the abdomen for measuring the down excursion of the spleen or liver (sophisticated teams can if they want look at the diaphragm).

At the lower BLUE-point, in a standard adult, a normal amplitude in quiet breathing is around $15+/-5 \mathrm{~mm}$. We speak of decreased lung sliding from the value of 4 to 10 $\mathrm{mm}$, quite abolished lung sliding between 1 and $3 \mathrm{~mm}$, and fully abolished at $0 \mathrm{~mm}$. Ultrasound is a very precise tool, and can detect a millimetric dynamic (even antique machines).

\section{One Basic Clinical Outcome: Lung Sliding and Pneumothorax}

For didactic reasons, we mention here one main application. A lung sliding, even very decreased, even not far from zero, allows pneumothorax to be ruled out [6].

For better understanding the ultrasound semiotics of pneumothorax, we advise to scan a patient with a complete pneumothorax. In these very didactic conditions, we will see first an absolute abolition of lung sliding, a major sign, but far from specific (the number of causes of abolished lung sliding is impressive, pleural adhesions among many others). We will see then the constant presence of A-lines (never B-lines). This association, called the A'profile when seen at the anterior wall (in supine patients) is here seen all over the chest wall. Note that when lung sliding is abolished, the Keye's space and the Merlin's space appear as two superimposed, strictly similar rectangles. The lung point will not be evoked in this review for keeping it short.

\section{Basic Other Clinical Outcomes - Analysis of Lung Ampliation, i.e., Assessment of Acute Respiratory Distress Syndrome (ARDS) -}

In the daily routine of the intensivist, lung sliding is a logical indicator of the lung ampliation. Measuring at the lower BLUE-point the amplitude of lung sliding provides a quantitative approach, from zero to roughly $20 \mathrm{~mm}$.

Lung sliding in standard workshops is easy to study (when the correct machine with the correct filters, i.e., no filter, are present) and all attendants are usually happy. In the real life, the intensivist will see either dyspneic patients, or sedated patients, generating two opposed patterns.

\section{1) The variant called "hectic variant"}

Dyspneic patients are challenging, because they are agitated, and mostly the accessory respiratory muscles are recruited, resulting in parasite dynamics visible in the Keye's space (Figure 4). This can be an issue for novices. The difficulty is highly decreased by the use of the M-mode, provided the machine is smart, i.e., with the M-mode not only side-by-side with the real-time, but exactly side by side, a simple feature which existed in the 1982 technologies but is surprisingly absent from most 


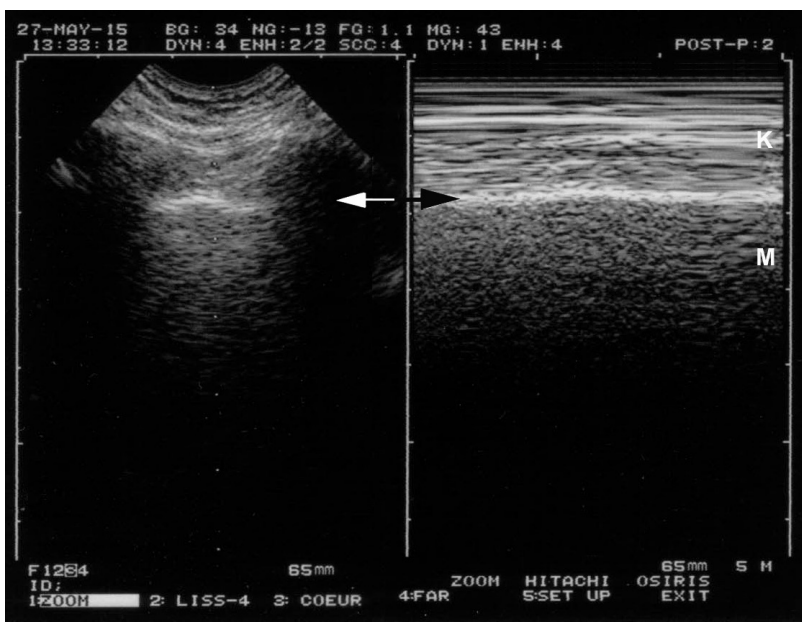

Figure 4. Lung sliding and dyspnea. On a frozen image, one can see that this patient is dyspneic. The left image allows localization of the pleural line (arrow). This location is reported at the right, allowing precise identification of the pleural line on $\mathrm{M}$ mode (arrow). Keye's space is not as homogeneous as in Figure 1 , as artifacts are caused by the recruitment of accessory respiratory muscles. Note however that the patterns of the two areas (Keye's and Merlin's spaces) are different, and lung sliding is easily identifiable.

modern laptop machines. This point must be taken into consideration when affording a new machine.

\section{2) The "minimal variant"}

In sedated, curarized patients, with protective ventilation (low tidal volume), on the opposite, there is no parasite dynamic, but lung sliding can be very discreete. Very subtle signs (called the grain of sand variant, the T-line) must be sought for. Figure 5 gives a clue for knowing that lung sliding is present or really abolished. The simplest technology without any filter is the best warrant to give a chance to this subtle semiotics to express itself (subtle but standardized). One question comes often, since many users have the feeling to see a lung point (i.e., a pneumothorax), when there is an alternance of seashore and stratosphere sign. We had to give a name to this phenomenon that was the mangrove variant (no matter why). The mangrove variant is explained by the post-inspiratory and post-expiratory pauses. No more lung sliding, no dynamic, and the seashore sign vanishes. The solution is standardized, just a bit long to explain. First, the pattern on M-

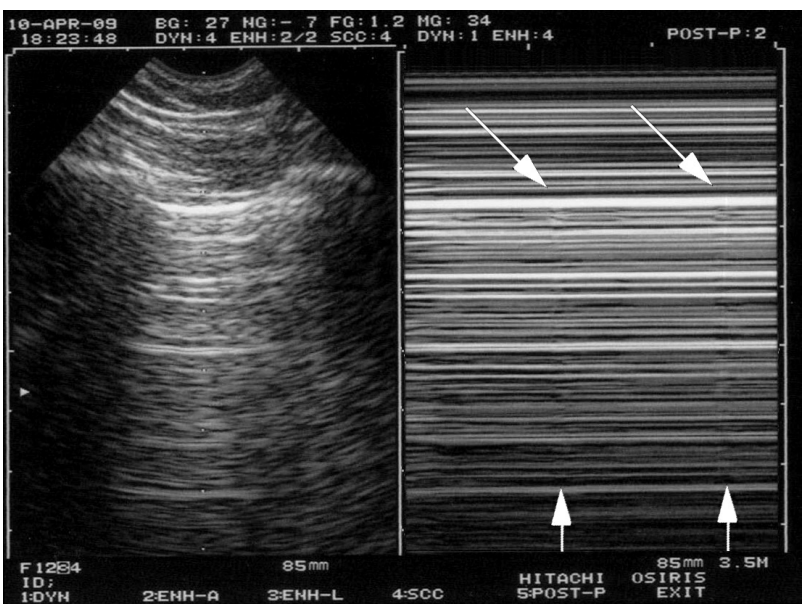

Figure 5. Extremely weak lung sliding in ventilated patients. The concept of the T-lines shows how subtle lung ultrasound can be. In the first view, this double image indicates pneumothorax: A-lines with a stratosphere sign. In reality, this is not a pneumothorax. This image is of a sedated patient with protective ventilation (low tidal volume); at the upper BLUE-point, all conditions for making lung sliding very discrete. The indications that the finding is not truly a pneumothorax are first because the image is not sufficient: if there is no lung point, it is too early to speak of pneumothorax. Second and most importantly, the oblique arrows show extremely subtle artifacts arising from the pleural line, and the vertical arrows show something like a vertical line (oblique arrows for not spoiling the subtle pattern). These are the T-lines and are an equivalent of the lung pulse. This extreme case shows that lung ultrasound can be compared with the subtle semiotics of ECG when a ventricular tachycardia is sought. BLUE: bedside lung ultrasound in emergency; ECG: electrocardiogram.

mode is smooth, not sudden like a genuine lung point. Second, if only the real-time is seen, no sudden change, but smooth changes in the speed of lung sliding are seen, in other words, the mangrove variant is created by the Mmode (maybe the only case where M-mode can confuse a bit). Third, the mangrove variant is seen on all points of the thorax, whereas the lung point is seen at a precise point (or line more exactly, but one point is enough for diagnosing pneumothorax). Fourth, for very worried users, a confusion (with a lung point) would make no clinical harm, because a (genuine) lung point seen all over the chest wall would mean a very minute pneumothorax, which would never explain the clinical concern (desaturation, e.g.) for which the patient benefits from an ultrasound. 


\section{How Lung Sliding Is Integrated in the Main CEURF Protocols: BLUE-protocol, FALLS- protocol, SESAME-protocol and the LUCIFLR Project}

The study of lung sliding is central to these protocols.

The BLUE-protocol aims at providing a bedside diagnostic approach to a respiratory failure [6]. Associations of signs are used (Figure 6). The A, B, A', A/B, $\mathrm{B}$ ' and C-profiles are all defined at the anterior wall of supine patients (principle $\mathrm{N}^{\circ} 2$ of LUCI). The A-profile associates normal lung sliding with A-lines (at this step, pulmonary embolism, asthma, chronic obstructive pulmonary disease (COPD) and some pneumoniae can be suggested, see the development below). The B-profile associates normal lung sliding with lung rockets; it likely indicates hemodynamic pulmonary edema. The B'-profile associates completely or quite completely abolished lung sliding with lung rockets; it highly suggests pneu- monia or ARDS. The C-profile describes anterior lung consolidations (regardless number or volume); it highly suggests pneumonia or ARDS. The A'-profile associates completely abolished lung sliding with A-lines; it suggests pneumothorax.

The A-profile generates 3 profiles allowing distinction. In the case of an A-profile, a venous assessment is done. As soon as a deep vein thrombosis (DVT) is detected, this is the A-DVT profile; the diagnosis of pulmonary embolism is more than highly suggested, with a $99 \%$ specificity (and a $81 \%$ sensitivity). If no DVT is found (sequential analysis, under submission), PLAPS are sought for. If they are found, the profile is the A-VPLAPS-profile (this term clearly indicates, A-profile, then venous analysis done but negative, then positive search for PLAPS); it indicates likely pneumonia or some ARDS. If PLAPS are missing, one speaks of the nude profile (A-profile, no DVT, no PLAPS), which is likened, by default, to severe asthma or COPD. Detailed

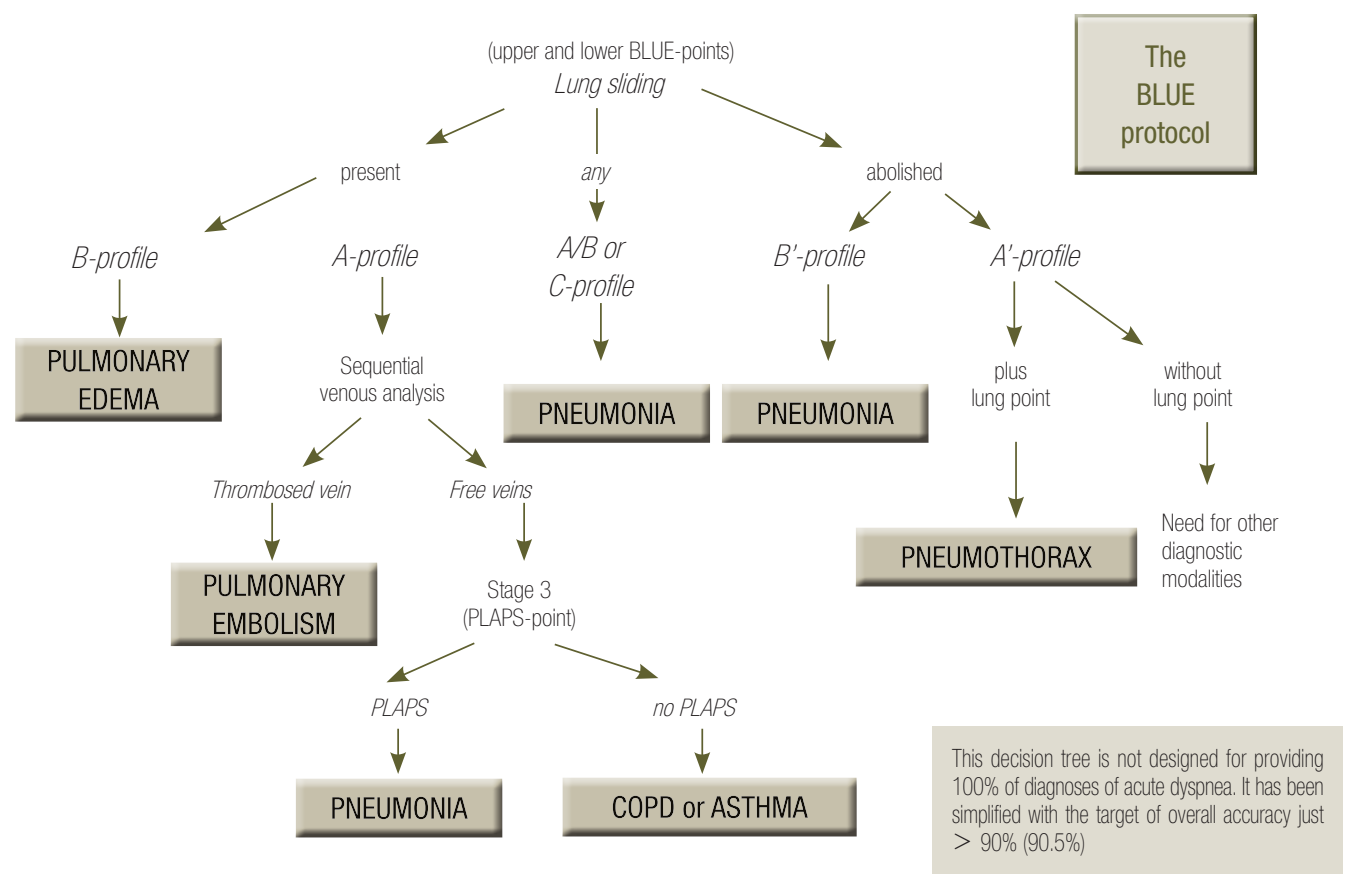

Figure 6. BLUE-protocol decision tree. This decision tree may appear complex, but the investment is worth it, since it provides the main diagnoses of acute respiratory failure with high accuracy. For going more in details, a whole book was necessary [3], but this decision tree makes a nice initial basis. One can see the lung sliding, at the very top. On an initial scan, abolished lung sliding might immediately suggest pneumothorax if it is associated with A-lines (the A'-profile) or pneumonia or ARDS if it is associated with lung rockets (the B'profile). Adopted from reference [6]. ARDS: acute respiratory distress syndrome; BLUE: bedside lung ultrasound in emergency; PLAPS: posterolateral alveolar and/or pleural syndrome. 
accuracy of the BLUE-protocol features in Table 1, for these six main diseases seen in adults visited in the emergency room and admitted to the intensive care unit, these six diseases making $97 \%$ of all patients.

The FALLS-protocol (fluid administration limited by lung sonography) in a very few lines. In an acute circulatory failure of unknown cause, a first look to the pericardium, then the right ventricle volume, then on lung sliding informs on pericardial tamponade, pulmonary embolism and pneumothorax. If the pericardium is dry, the right ventricle nondilated, and lung sliding present, obstructive shock can reasonably be ruled out. The absence of a B-profile shows that a cardiogenic shock from left origin can be reasonably ruled out. The patient is then decreed "FALLS-responder", because the two remaining causes of shock are hypovolemic and distributive, and both require fluids. Fluid is given at this step, under the control of clinical/biological signs of improvement. A hypovolemic shock, schematically, resolves under fluid therapy. If no improvement occurs, there is no signal for discontinuing fluid therapy, up to the point that the interstitial part of the lung gets filled, generating B-lines, i.e., the interstitial step of pulmonary edema. This step is clinically and biologically occult. Distributive shock can reasonably be evoked at this step, i.e., in daily routine, septic shock. This is a very schematical approach, many questions are answered in our textbook [3].

The SESAME-protocol invites to assess the cause of a cardiac arrest (pinpointing in few dozens of seconds on the main reversible causes, shockable causes apart). Ultrasound (we mean, using traditional machines) should never be used for managing a cardiac arrest. The diagnosis must be clinical. This being said, the lucky ones who have our equipment will be highly glad to find there: a narrow (not especially laptop) machine of 32-cm width, which switches on in 7 seconds, no button to touch (the machine is always ready for the SESAME-protocol), and only one probe for this whole body approach. This begins by pneumothorax (with the analysis of lung sliding), then pulmonary embolism (adapting the BLUE-protocol by searching for a DVT at a specific location), then abdominal bleeding, then pericardial tamponade. The very heart comes 5 th, because most reversible causes have been diagnosed before this step.

LUCIFLR project. LUCI can favor limitation of radiations (LUCIFLR). The LUCIFLR project aims at decreasing the number of urgent bedside radiographs by $1 / 3$, and the number of urgent chest CT by $2 / 3$, in the 3 next decades; these are reasonable targets. Given that the negative predictive value of abolished lung sliding is $100 \%$, the vision of lung sliding in a patient with: suspicion of pneumothorax, management of pneumothorax,

Table 1. Accuracy of the BLUE-protocol

\begin{tabular}{|c|c|c|c|c|c|}
\hline Origin of dyspnea & Profiles of BLUE-protocol & Sensitivity (\%) & Specificity (\%) & $\begin{array}{c}\text { Positive predictive } \\
\text { value (\%) }\end{array}$ & $\begin{array}{c}\text { Negative predictive } \\
\text { value (\%) }\end{array}$ \\
\hline Acute hemodynamic pulmonary edema & B-profile & 97 & 95 & 87 & 99 \\
\hline \multirow[t]{5}{*}{ Pneumonia (four profiles) } & B'-profile & 11 & 100 & 100 & 70 \\
\hline & A/B profile & 14.5 & 100 & 100 & 71.5 \\
\hline & C-profile & 21.5 & 99 & 90 & 73 \\
\hline & A-V-PLAPS profile & 42 & 96 & 83 & 78 \\
\hline & The four profiles & 89 & 94 & 88 & 95 \\
\hline $\begin{array}{l}\text { Exacerbated COPD or severe acute } \\
\text { asthma }\end{array}$ & Nude profile & 89 & 97 & 93 & 95 \\
\hline Pulmonary embolism & $\begin{array}{l}\text { A-profile (with deep venous } \\
\text { thrombosis) }\end{array}$ & 81 & 99 & 94 & 98 \\
\hline Pneumothorax & A'-profile (with lung point) & 88 & 100 & 100 & 99 \\
\hline
\end{tabular}

Modified from reference [6]. Table modified, for displaying the diseases in function of their frequency.

BLUE: bedside lung ultrasound in emergency; PLAPS: posterolateral alveolar and/or pleural syndrome; COPD: chronic obstructive pulmonary disease. 
routine when a pneumothorax can occur such as mechanical ventilation (etc.), allows to postpone irradiating tests. We still fight today for decreasing the use of CT when doctors want to rule out pneumothorax by any means (before airplane transportation e.g.). Just for this application, the cost-savings and radiations savings can be huge. When the whole of LUCI (and critical ultrasound) is exploited, this generates a disruptive cost saving. Any doctor who, in his or her practice, postpones a bedside radiograph when the only question is pneumothorax and when ultrasound clearly answered no, performs, aware or not of this, the LUCIFLR project.

\section{A Last Comment}

The ultrasound vision of lung sliding may appear a remarkable sign, because it gives to the user the vision of a whole lung which is sliding, with no efford, absolutely no pain, no sensation linked to a rubbing. Like the heart, like the systolic expansion of the arterial blood through the whole body, the human factory works without noise, when it works.

\section{Conclusions}

Lung sliding must be accepted as a reasonable gold standard.

LUCI, not supposed to exist, is the tool for a visual medicine. A standardized field, it provides this daily vision of the inside, and should reach the interest of the intensivist as well as many specialties: briefly all the ones currently using the stethoscope (anesthesiology, prehospital medicine, pediatric intensive care, emergency medicine, and also internal medicine, family medicine and some others). Lung sliding, among many multifaceted applications, can solve issues about irradiation (and cost issues in all these countries which are interested in making savings; colleagues should just focus on the principle $\mathrm{N}^{\circ} 1$ : simple equipment, single probe mainly). Lung sliding, a small part of LUCI, itself a small part of critical ultrasound, itself a small part of ultrasound, should be seen, more than a data, also a bit of a philosophy [7].

\section{Acknowledgements}

Videos are available at www.CEURF.net, section BLUE-protocol, with examples of 1) a normal lung sliding in the A-profile, 2) a conserved lung sliding in the Bprofile, 3) abolished lung sliding with pneumothorax in the A'-profile, 4) abolished lung sliding with ARDS in the B'-profile.

\section{ORCID}

Daniel A. Lichtenstein http://orcid.org/0000-0002-6923-1056

\section{References}

1. Friedman PJ. Diagnostic procedures in respiratory diseases. In: Harrison's principles of internal medicine. 12th ed. New York: McGraw-Hill; 1992. p 1043.

2. Lichtenstein DA. Lung ultrasound in the critically ill. Ann Intensive Care 2014; 4; 1.

3. Lichtenstein DA. Lung Sliding. In: Lung Ultrasound in the Critically Ill - the BLUE-protocol. 6th ed. Heidelberg: Springer-Verlag International; 2016. pp 67-78.

4. Lichtenstein DA, Mezière GA. The BLUE-points: three standardized points used in the BLUE-protocol for ultrasound assessment of the lung in acute respiratory failure. Crit Ultrasound J 2011; 3: 109-10.

5. Guyton AC, Hall JE. Textbook of medical physiology. 9th ed. Philadelphia: W.B. Saunders Company; 1996. pp 496-7.

6. Lichtenstein DA, Mezière GA. Relevance of lung ultrasound in the diagnosis of acute respiratory failure: the BLUE-protocol. Chest 2008; 134: 117-25.

7. van der Werf TS, Zijlstra JG. Ultrasound of the lung: just imagine. Intensive Care Med 2004; 30: 183-4. 